\title{
Retraction of: miR-106a-5p Suppresses the Proliferation, Migration, and Invasion of Osteosarcoma Cells by Targeting HMGA2, by Qian-Yun He, et al. DNA Cell Biol, 35(9) 506-520; DOI: 10.1089/dna.2015.3121
}

DNA and Cell Biology officially retracts the paper entitled, "miR-106a-5p Suppresses the Proliferation, Migration, and Invasion of Osteosarcoma Cells by Targeting HMGA2," by Qian-Yun He, Guang-Chao Wang, Hao Zhang, Da-Ke Tong, Chen Ding, Kang Liu, Fang Ji, Xiongbai Zhu, and Shengwu Yang. DNA Cell Biol, 35(9) 506-520; DOI: 10.1089/ dna.2015.3121, due to suspicion of scientific misconduct.

The corresponding author, Dr. Fang Ji, contacted the journal via email, and requested a correction be made to Figure 8A, indicating, "When I recently collated the results of the project, I found that in the immunofluorescence experiment, as I used several folders to store pictures but the naming was not standardized, the pictures of other groups in Figure 8A scramble group, hmega2 siRNA group, mir-106a-5p + hmega2 group were misused. [sic]"

The email also contained a new figure, however, upon examination by the Editor, it was clear that there were differences between the published figure and the replacement figure. There was clear duplication of lanes in the Western blot figure. The author was then asked to provide further explanation, and Dr. Ji's response was, "Figure 8 A scramble group, hmega2 siRNA group and mir-106a-5p + hmega2 group used the wrong pictures of other groups, and provided the three groups of pictures again [sic]." This response did not address the confusion and several other requests for clarification were made, but went unanswered. The lack of response for clarification concerned the Editor who then re-reviewed the request again, which resulted in the discovery that additional problematic images were raised on the PubPeer platform ${ }^{1}$ and believes that the authors are now eager to correct them as there appears to be clear scientific misconduct with respect to digital manipulation of figures. Considering the available evidence and lack of further details from the authors, the Editor officially retracts the article from the journal.

The Editor and Publisher of DNA and Cell Biology are committed to preserving the scientific literature and the community it serves.

\section{Reference}

1. https://pubpeer.com/publications/35F4FADC69A2182180559AC68655CE Last accessed September 3, 2020. 